TTR

Traduction, terminologie, rédaction

\title{
« Nous les femmes " de 1970 à 2017 à travers les traductions et adaptations de Our Bodies, Ourselves en français
}

\section{Nesrine Bessaïh et Anna Bogic}

Volume 29, numéro 2, 2e semestre 2016

Translation and Power: Countertactics

La traduction et le pouvoir : la contre-tactique

URI : https://id.erudit.org/iderudit/1051013ar

DOI : https://doi.org/10.7202/1051013ar

Aller au sommaire du numéro

\section{Éditeur(s)}

Association canadienne de traductologie

ISSN

0835-8443 (imprimé)

1708-2188 (numérique)

Découvrir la revue

\section{Citer cet article}

Bessaïh, N. \& Bogic, A. (2016). « Nous les femmes » de 1970 à 2017 à travers les traductions et adaptations de Our Bodies, Ourselves en français. TTR, 29(2), 43-71. https://doi.org/10.7202/1051013ar

\section{Résumé de l'article}

Le présent article se penche sur les traductions et adaptations françaises du classique féministe Our Bodies, Ourselves (OBOS) publié aux États-Unis en 1971. Nous explorons la diversité du "nous » dans l'expression « nous les femmes » à travers trois traductions et adaptations de $O B O S$ réalisées dans des environnements francophones qui diffèrent par l'époque et la situation géopolitique. Nous postulons que ces projets de traduction témoignent des tentatives de représentations de la diversité du « nous » en recourant à la réflexivité et l'intersectionnalité, deux approches qui invitent les traductrices à réfléchir à leur propre posture vis-à-vis des autres femmes et à reconnaître les rapports de pouvoir inégaux qui peuvent exister entre elles. Dans la première partie de l'article, nous présentons les origines de $O B O S$ avant de nous pencher sur les versions françaises réalisées respectivement en France et au Sénégal. Dans la deuxième partie, nous traitons de l'intersectionnalité, de son développement au sein du mouvement des femmes au Québec et de son rôle clé dans le processus d'adaptation adopté par la collective de militantes qui travaille actuellement au Québec à une nouvelle adaptation de $O B O S$. L'engagement des traductrices envers la réflexivité et l'intersectionnalité montre leur volonté de prendre en considération les rapports de pouvoir inégaux entre des femmes qui occupent différentes postures face au «nous les femmes ». De plus, le cas de OBOS illustre une approche de la traduction où la fidélité ne se mesure pas à l'aune de la retransmission exacte du texte; il s'agit plutôt d'une approche redevable au message et au projet politique d'une reprise de pouvoir des femmes sur leur vie. 


\title{
"Nous les femmes» de 1970 à 2017 à travers les traductions et adaptations de Our Bodies, Ourselves en français
}

\author{
Nesrine Bessaïh et Anna Bogic
}

Université d'Ottawa

\begin{abstract}
Résumé
Le présent article se penche sur les traductions et adaptations françaises du classique féministe Our Bodies, Ourselves (OBOS) publié aux États-Unis en 1971. Nous explorons la diversité du «nous» dans l'expression «nous les femmes» à travers trois traductions et adaptations de $O B O S$ réalisées dans des environnements francophones qui diffèrent par l'époque et la situation géopolitique. Nous postulons que ces projets de traduction témoignent des tentatives de représentations de la diversité du «nous» en recourant à la réflexivité et l'intersectionnalité, deux approches qui invitent les traductrices à réfléchir à leur propre posture vis-à-vis des autres femmes et à reconnaître les rapports de pouvoir inégaux qui peuvent exister entre elles. Dans la première partie de l'article, nous présentons les origines de $O B O S$ avant de nous pencher sur les versions françaises réalisées respectivement en France et au Sénégal. Dans la deuxième partie, nous traitons de l'intersectionnalité, de son développement au sein du mouvement des femmes au Québec et de son rôle clé dans le processus d'adaptation adopté par la collective de militantes qui travaille actuellement au Québec à une nouvelle adaptation de OBOS. L'engagement des traductrices envers la réflexivité et l'intersectionnalité montre leur volonté de prendre en considération les rapports de pouvoir inégaux entre des femmes qui occupent différentes postures face au «nous les femmes». De plus, le cas de OBOS illustre une approche de la traduction où la fidélité ne se mesure pas à l'aune de la retransmission exacte du texte; il s'agit plutôt d'une approche redevable au message et au projet politique d'une reprise de pouvoir des femmes sur leur vie.
\end{abstract}

Mots-clés: Our Bodies Ourselves, santé des femmes, adaptation culturelle, réflexivité, intersectionnalité

Abstract

The focus of the present article is on the French-language translations and adaptations of Our Bodies, Ourselves (OBOS), a classic of feminist literature 
published in the United States in 1971. We explore the diversity implicit in the "we" and "us" that inform expressions like "we women" or "us women" by examining three translations and adaptations of $O B O S$ produced in historically and geopolitically different francophone environments. These translations, we will argue, demonstrate an attempt to represent this diversity through research and writing methods informed by reflexivity and intersectionality, both of which invite women translators to reflect on their own position vis-à-vis the other women they are writing about, and to acknowledge the unequal power relationships that may exist between them. In the first part of the article, we discuss the origins of $O B O S$ and then the French-language versions published in France and in Senegal. In the second part, we focus on intersectionality, its development within the women's movement in Quebec, and its key role in the adaptation process undertaken by a group of feminist activists currently working on the new translation of OBOS. By engaging with reflexivity and intersectionality, the translators demonstrate a willingness to take into consideration unequal power relations between women who are differently positioned vis-à-vis "we women." Further, the case of $O B O S$ offers a translation approach where "faithfulness" lies not in the literal retransmission of the source text, but rather in upholding the message of a political project aimed at helping women regain power over their lives.

Keywords: Our Bodies Ourselves, women's health, cultural adaptation, reflexivity, intersectionality

\section{Introduction}

Après près de cinquante ans de luttes féministes, de manifestations et de revendications pour l'égalité, le mouvement des femmes cherche plus que jamais des façons de se consolider. Issue du féminisme des années 1970, l'expression «nous les femmes» a réussi à unir les femmes et à soutenir leur prise de parole pour réclamer l'égalité dans toutes les sphères de la vie. Cependant, au fil des années, il est devenu évident que si le mouvement voulait maintenir sa pertinence et son impact politique, il allait devoir remettre en question les différences et les inégalités entre les femmes elles-mêmes. Dès les années 1970, les féministes françaises font intervenir dans leurs analyses les enjeux liés aux classes sociales tandis qu'aux États-Unis, les féministes afroaméricaines réfléchissent aux incidences de la classe sociale, de la couleur de la peau et de l'orientation sexuelle sur les conditions de vie des femmes (Fassin, 2015). À la fin des années 1980, le féminisme intersectionnel cherche à reconnaître les formes de discrimination auxquelles les femmes font face et qui façonnent des vécus fort différents les uns des autres (Crenshaw, 2005 
[1991]; Davis, 2015). Parallèlement, la santé des femmes, et surtout la santé sexuelle et reproductive, demeure une des pierres angulaires des luttes féministes.

Aux États-Unis, Our Bodies, Ourselves $(O B O S)$, un livre phare sur la santé des femmes publié pour la première fois en 1971, contribue au développement d'un véritable mouvement pour la santé des femmes (Morgen, 2002). Le livre traverse rapidement les frontières; des dizaines d'associations de femmes se l'approprient, car elles y reconnaissent un besoin quelles partagent. OBOS a depuis été traduit et adapté dans plus de trente langues et a mis en relation de nombreuses associations de femmes à travers le monde (Davis, 2007). Une des caractéristiques fondamentales de $O B O S$ réside dans l'usage qui y est fait du pronom de la première personne du pluriel, «we» («nous»), qui se veut un appel lancé à toutes les femmes pour qu'elles se joignent au mouvement féministe. Et pourtant, la diversité de ce «nous» continue de poser des défis au mouvement, qui semble constamment menacé d'imploser sous l'effet des revendications de reconnaissance des identités multiples et des oppressions vécues. Alors que $O B O S$ embrasse différents contextes, lieux géopolitiques et périodes historiques, ce «nous» utilisé par les traductrices de l'ouvrage se transforme aussi.

Nous proposons d'explorer la diversité du «nous» dans l'expression "nous les femmes" à travers trois traductions et adaptations françaises de $O B O S$ réalisées dans des environnements francophones qui diffèrent par l'époque et la situation géopolitique. Nous postulons que ces traductions témoignent des tentatives de représentations de la diversité du «nous» en recourant à la réflexivité et à l'intersectionnalité, deux approches qui invitent les traductrices à réfléchir à leur propre posture vis-à-vis des autres femmes et à reconnaître les rapports de pouvoir inégaux qui peuvent exister entre elles. En utilisant le pronom de la première personne du pluriel, les auteures du texte source de même que les traductrices contribuent à la création d'un espace imaginaire dans lequel une identité partagée serait possible. Le "nous", en tant quélément discursif, interpelle ainsi les lectrices et les invite à joindre les mouvements féministes. Il constitue un acte perlocutoire ${ }^{1}$, c'est-à-dire un acte de parole qui provoque l'action en incitant les lectrices à agir. En plus de ce «nous» de $O B O S$,

1. Sur la notion de traduction perlocutoire, voir Brisset (1990). 
nous, les auteures du présent texte, utiliserons le même pronom pour nous désigner. Ajoutons que, pour répondre à un principe de réflexivité, nous sommes deux femmes cis canadiennes de classe moyenne, immigrantes, allophones, féministes engagées travaillant dans le domaine de la traductologie au sein d'une université canadienne.

Dans la première partie de l'article, nous présenterons les origines de $O B O S$ avant de nous pencher sur la version française parue en France en 1977 et sur la version sénégalaise parue en 2004. Nous traiterons ensuite, dans la deuxième partie, de l'intersectionnalité, de son développement au sein du mouvement des femmes au Québec et de son rôle clé dans le processus d'adaptation adopté par la collective de militantes qui travaille actuellement à une nouvelle traduction française de $O B O S$.

Notre réflexion s'appuie sur des données recueillies lors d'entretiens semi-dirigés réalisés avec des traductrices et coordonnatrices des traductions ${ }^{2}$ ainsi qu'à partir d'une analyse comparative des traductions et du texte source. Nous avons de plus effectué une analyse approfondie du contenu préfaciel du texte source et des trois traductions examinées, ainsi que de textes secondaires relatifs au contexte politique et historique dans lequel s'inscrit chacune des traductions, en particulier les textes portant sur le contexte propre au mouvement de libération des femmes.

Nous porterons une attention particulière à la notion de fidélité en traduction et nous soutiendrons que $O B O S$ échappe à la compréhension traditionnelle de cette notion. De fait, la notion de fidélité a dès le départ été remise en question dans les rangs des traductrices féministes, en raison notamment du contexte dans lequel la traduction féministe a pris naissance. Marquées par le mouvement de libération des femmes et l'essor des théories féministes, les années 1970 et 1980 sont aussi, au Québec, influencées par le mouvement indépendantiste. Inspirées par cette

2. Nous avons rencontré trois femmes qui ont participé à la traduction produite en France en 1977, soit Nicole Bizos-Cormier, Sophie Mayoux et Lyba Spring. Les entretiens ont été réalisés en juin et en septembre 2015. Nous avons par ailleurs réalisé en août 2015 un entretien avec Codou Bop, qui a participé à l'élaboration de la version sénégalaise. Enfin, le projet de traduction qui est en cours au Québec est étudié par l'une des auteures du présent article, Nesrine Bessaïh, qui est impliquée au sein de l'organisme qui coordonne la traduction. La méthodologie repose sur l'observation participante ainsi que sur l'analyse des documents produits par la collective de traductrices et adaptatrices. 
période d'effervescence, des auteures québécoises développent une écriture expérimentale dite d'avant-garde par laquelle elles cherchent à rendre le féminin visible et à déconstruire le langage patriarcal. L'apparition de l'École canadienne de traduction féministe est intimement liée à l'entreprise de traduction de ces auteures québécoises. Comme lobserve Luise von Flotow (1997), traduire des textes expérimentaux nécessite une pratique féministe et subversive de la traduction. Pour les traductrices féministes, le sens n'est jamais ni transparent ni une simple représentation d'un texte «original». Au contraire, la traduction féministe exige des interventions et des pratiques novatrices afin de questionner puis de transformer la langue (patriarcale) dominante (Maier, 1985; Godard, 1989; Simon, 1996; von Flotow, 1997; Ergun, 2015)33.

Les travaux de Kathy Davis sur OBOS et ses traductions, publiés en 2007, constituent une contribution majeure aux études sur la traduction féministe, puisqu'ils mettent en évidence le rôle de la traduction dans la production des savoirs féministes dans différents contextes socioculturels. Gayatri Chakravorty Spivak soulève pour sa part un enjeu particulièrement important dans son article «Politics of Translation» (2004 [1992]), soit le fait que la solidarité entre femmes ne peut se construire sans la reconnaissance des rapports de pouvoirs inégaux entre les langues parlées par les femmes du Premier Monde et celles parlées par les femmes du Tiers Monde. Ester Shapiro (2013) insiste de son côté sur l'ancrage des pratiques de traduction dans les pratiques militantes locales. Le présent article s'inscrit dans la foulée de ces travaux, en abordant la mise en œuvre de théories et de pratiques sociales militantes et féministes pour produire des savoirs féministes (dans notre cas, la réflexivité dans les années 1970 et l'intersectionnalité dans les années 2010). D’ailleurs, nous soutenons que le succès des traductions de $O B O S$ repose sur leur capacité à s'adapter à de nouveaux contextes et à tenir compte des différentes conditions de vie qui entraînent une pluralité de «nous les femmes». En adoptant une posture qui favorise la réflexivité et l'intersectionnalité, les traductrices de $O B O S$ démontrent en effet leur volonté de prendre

3. Chamberlain (1988) et Lotbinière-Harwood (1991) font également partie des textes clés sur la traduction féministe. Après une période relativement calme au début des années 2000, le sujet suscite de nouveau l'intérêt. Pour un survol de travaux récents, on peut se référer à Susam-Sarajeva (2006), Federici (2011), von Flotow (2011), von Flotow et Farahzad (2016), et Castro et Ergun (2017). 
en considération les rapports de pouvoir inégaux qui existent entre les traductrices et les lectrices, entre des femmes qui occupent différentes postures face au "nous les femmes». Ce faisant, elles adoptent une approche de la traduction où la fidélité ne se mesure pas à l'aune de la retransmission exacte du texte; l'approche qu'elles privilégient est plutôt redevable au message et au projet politique d'une reprise de pouvoir des femmes sur leur vie. Comme l'a montré Kathy Davis, ce qui voyage ou ce qui est transférable à travers $O B O S$ ne tient pas tant au contenu informationnel du texte source ni à une supposée identité partagée de femmes, mais à une méthode de partage des savoirs qui rend possible l'avènement d'une solidarité féministe transnationale entre des femmes aux statuts sociaux variés et inégaux (2007, p. 79). Les traductions de $O B O S$ en français offrent ainsi une occasion unique de retracer les transformations du «nous» au sein du projet politique féministe, de 1970 à nos jours.

\section{Our Bodies, Ourselves et son parcours transnational}

Issu du mouvement pour les droits civiques, des mobilisations antiguerre et de la Nouvelle Gauche américaine, le mouvement de libération des femmes a dénoncé au fil des années de nombreuses pratiques oppressives vécues par les femmes : violence conjugale, harcèlement sexuel, criminalisation de l'avortement, lois injustes encadrant le divorce, discrimination sur le marché du travail et dans le système scolaire. En même temps que les militantes de ce mouvement organisaient des manifestations, elles ont entrepris de construire un discours féministe radical dans lequel le corps est devenu un symbole de libération. À travers des groupes de conscientisation, des femmes se sont rassemblées pour partager leurs expériences et leurs histoires les plus intimes et ont formulé le slogan le plus célèbre que l'histoire a gardé de cette période : «le privé est politique».

C'est en 1969, en pleine période de bouleversements sociaux et politiques aux États-Unis, qu'une douzaine de femmes participant à une conférence sur la libération des femmes décident de mettre sur pied le Boston Women's Health Book Collective (appelé le collectif de Boston ci-après). Elles se fixent alors pour objectif d'élaborer un manuel radical d'auto-santé pour répondre à leur désir criant de prendre le contrôle de leur corps et de leur santé reproductive (Davis, 2007). Publié en 1971 sous le titre Our 
Bodies, Ourselves, l'ouvrage contient de l'information médicale vulgarisée, des témoignages de femmes et le récit d'expériences trop souvent pénibles vécues par les femmes. ${ }^{4} \mathrm{Il}$ fournit de l'information sur l'anatomie et la sexualité, l'homosexualité, la masturbation, la violence faite aux femmes, les relations intimes, l'auto-santé, l'auto-défense, la contraception (incluant la pilule anticonceptionnelle, fort controversée à l'époque), l'avortement (alors illégal ${ }^{5}$ ), la grossesse, l'accouchement et le vieillissement. On y trouve également des photographies et des illustrations de vrais corps de femmes, ce qui donne aux lectrices des connaissances essentielles et émancipatrices sur des sujets jusquelà tabous, rarement discutés sauf en termes honteux et coupables. Le texte est hautement politisé; il aborde les lectrices sur un ton qui les interpelle et qui les invite à se joindre au mouvement des femmes.

Leffet de $O B O S$ est immédiat et son impact est à la mesure du traitement que la société américaine de l'époque réserve au corps féminin et à la sexualité :

There was virtually no open discussion of sex and reproduction in schools or the popular media, and physicians condescended to women and regularly withheld medical information from their female patients. (Gordon et Thorne, 1998, p. 182)

L'engouement se révèle dans le nombre d'exemplaires vendus, soit plus de 200000 en quelques mois. Ce «succès alternatif» se répand surtout grâce au bouche-à-oreille et aux tout récents programmes d'études féministes (Sanford, 1979, p. 85). Dans son livre sur le mouvement pour la santé des femmes aux ÉtatsUnis, Sandra Morgen soutient, au sujet de l'influence exercée par $O B O S$ : «it would be difficult to exaggerate the impact of Our Bodies, Ourselves [...]. Erupting into the void (there were few popular books about women's health before it), Our Bodies, Ourselves created its own niche» (2002, p. 19).

4. Une première version intitulée Women and Their Bodies, imprimée sous forme de brochure, est parue en 1970 (cf. Our Body Ourselves, s.d.).

5. Aux États-Unis, l'avortement a été légalisé au niveau fédéral en 1973, après le procès Roe vs Wade. Toutefois, plusieurs États (parmi lesquels la Californie, le Colorado, le Maryland et New York) avaient intégré des réformes dès 1969 et au début de 1970 pour permettre des "avortements thérapeutiques", ce qui rendait la procédure plus accessible. Les premières éditions de OBOS expliquaient en détail les lois sur l'avortement (BWHBC, 1970, 1973, 1976). 
Neuf éditions de $O B O S$ ont été publiées à ce jour, respectivement en 1971, 1973,1976,1979, 1984,1992, 1998,2005 et 2011. Le contenu et la forme du texte ont évolué, parallèlement aux cohortes et aux idées féministes. De nouvelles perspectives et de nouveaux concepts se sont ajoutés, par exemple, la justice reproductive et le vécu des personnes trans. Le ton a changé également : il reflète l'évolution terminologique et l'atténuation de la verve du féminisme radical si caractéristique des États-Unis des années 1960 et 1970. Depuis 1971, OBOS s'est vendu à plus de 4,5 millions d'exemplaires et a investi la culture populaire par la télévision, le cinéma et la littérature. Au fil des éditions, le collectif de Boston a maintenu son engagement envers le militantisme féministe et le florissant mouvement pour la santé des femmes ainsi que pour un processus collaboratif de production de savoirs. Chaque édition est le résultat du travail et de la collaboration de dizaines, voire de centaines de personnes ${ }^{6}$.

Au moment même où $O B O S$ se popularise aux États-Unis, des traductions et des adaptations commencent à faire leur apparition dans divers pays. Aujourd'hui, plus de trente traductions/ adaptations sont recensées par la Our Bodies Ourselves Global Initiative $(\mathrm{OBOGI})^{7}$, mise sur pied par le collectif de Boston afin de soutenir et de réseauter ses partenaires internationaux. On compte également près d'une douzaine de projets en cours de réalisation. Selon Kathy Davis, OBOS aurait connu trois vagues de diffusion : de 1970 à 1982 en Europe de l'Ouest et au Japon; de 1982 à 1996 en Asie, en Afrique et au Moyen-Orient; de 1996 à 2006 dans les pays dits en voie de développement et en Europe de l'Est (2007, p.52-53). Les approches de traduction et les versions produites varient considérablement : certaines versions sont de strictes traductions alors que d'autres sont de véritables adaptations culturelles. Dans certains cas, l'ouvrage original et son histoire épique ont simplement joué un rôle d'inspiration.

La multiplication des ouvrages consacrés à la santé des femmes et la popularisation d'Internet ont fait en sorte que $O B O S$ a graduellement perdu son pouvoir de transformation de la

6. Selon le site de OBOS (http://www.ourbodiesourselves.org/), plus de 500 personnes ont contribué à l'édition de 2011.

7. Les informations sur les traductions de $O B O S$ sont disponibles sur le site du collectif, dans la section "global projects» (https://www.ourbodiesourselves. org/global-projects/). 
société américaine. À mesure que $O B O S$ gagnait de l'importance à l'extérieur des États-Unis (alors qu'à l'intérieur il se fondait dans la masse de publications sur la santé des femmes), son parcours international s'est mis à peser plus lourd dans la balance. De fait, les traductions de $O B O S$ continuent de mettre en lumière le pouvoir transformationnel de l'original. Elles contribuent en outre à donner une nouvelle vie au texte source en créant un cercle vertueux qui bénéficie aux deux projets. La perte d'influence de $O B O S$ sur le plan local, aux États-Unis, est largement compensée par le nouveau rôle que l'ouvrage assume comme "facilitator of its life outside the United States»(Davis, 2007, p. 79; nous soulignons). Alors que la majorité des traductions de $O B O S$ sont des adaptations qui tiennent compte du contexte culturel, l'élément qui reste constant dans toutes les versions repose sur l'idée « of a small group of laywomen talking about their embodied experiences and critically assembling useful information about their health needs » (ibid.).

\section{La traduction française et la notion de réflexivité}

Dès sa publication, $O B O S$ a commencé à traverser les frontières, souvent littéralement entre les mains de militantes féministes américaines qui, dans les années 1970 et 1980, rendaient visite à des groupes féministes d'autres pays à l'occasion de rencontres et de congrès internationaux sur la santé des femmes. Des membres du collectif de Boston, Judy Norsigian et Norma Swenson en particulier, se sont notamment rendues à Rome en 1975 pour participer à des ateliers portant sur les cliniques féministes d'autosanté ainsi qu'aux réunions internationales «Femmes et Santé» (International Women and Health Meetings) tenues en Suisse, aux Pays-Bas et au Costa Rica (Kramarae et Spender, 2000, p. 927; Bracke, 2015, p. 567). À la même époque, le collectif de Boston reçoit régulièrement des demandes par courrier de groupes de femmes de partout dans le monde qui souhaitent obtenir des exemplaires gratuits de $O B O S^{8}$.

Dans la foulée de l'agitation politique et des manifestations étudiantes de mai 1968, la France représente un terrain propice au développement du Mouvement de libération des femmes (le

8. On trouve dans les archives du collectif de Boston à la Schlesinger Library de l'Université Harvard de nombreuses lettres dans lesquelles des exemplaires gratuits sont demandés, ce qui témoigne de l'immense popularité du livre et de sa réputation de «bible féministe» sur la santé des femmes. 
MLF) et du militantisme féministe (Pisan et Tristan, 1977). $\mathrm{Au}$ début des années 1970, inspirés par les cliniques féministes d'auto-santé de Los Angeles, Boston et New York notamment, des collectifs se lancent dans des initiatives similaires dans diverses régions de la France. Six militantes passionnées de santé des femmes, basées à Paris et engagées dans le MLF, découvrent $O B O S$ et décident de fonder un collectif de traduction à Paris en 1972. Comme l'explique une de ses membres, Nicole BizosCormier, la principale motivation du groupe à traduire $O B O S$ en français émanait d'un désir de combler le manque de connaissances relatives au corps et à la sexualité des femmes : «un désir par rapport à ce que moi j'avais vu... si on pouvait arriver à produire ce livre pour la France, pour les femmes, parce qu'il n'y avait pas d'équivalent, avec tous ces aspects-là »".

Le collectif travaille à la traduction et à l'adaptation culturelle du livre entre 1973 et 1975. Notre corps, nous-mêmes est finalement publié en 1977. Le collectif essaie d'abord de produire une traduction qui colle au texte source américain. Cependant, les membres se rendent rapidement compte qu'il manque à leur traduction une voix qui s'adresse aux femmes françaises, une voix cohérente qui exprime une pratique féministe engagée. Elles décident alors de changer leur approche et de se concentrer plutôt sur l'adaptation du texte, s'éloignant souvent du texte source. Dans ce cas-ci, la fidélité au texte source n'avait pas produit l'effet recherché : la traduction devait s'imprégner du contexte local afin de capter l'intérêt des lectrices françaises :

Nous nous sommes donc au début limitées à une pure et simple traduction. [...] Mais cette ambiance, ce style, caractéristiques d'un collectif de femmes américain, avec son langage, sa façon d'exposer et d'articuler les idées, correspondant à une pratique, et nous ne pouvions pas les communiquer par la simple traduction. Au bout d'un an, nous avons dû reconnaître que les chapitres que nous avions produits étaient pratiquement illisibles. (Collectif de Boston pour la santé des femmes, 1977, p. 11).

À partir du moment où les membres du collectif français se rendent compte qu'elles ne peuvent adopter le «nous» des féministes américaines, elles abandonnent l'idée d'être fidèles au texte source et de produire une traduction directe. Elles estiment

9. Entretien avec Nicole Bizos-Cormier, septembre 2015. 
que la traduction directe qu'elles ont réalisée au départ est indigeste pour les femmes françaises. Dans ce contexte, la fidélité prend forme autour d'un projet politique, soit le fait de reprendre possession de son corps et des savoirs qui le concernent. Ainsi, les membres du collectif français construisent une position discursive par leur analyse réflexive et se positionnent parmi les femmes françaises. Il devient dès lors difficile de traduire sans intervenir sur le texte et sans l'adapter ${ }^{10}$.

Sur le plan idéologique, le collectif de traduction partage les positions du MLF ainsi que de la gauche communiste. Nicole Bizos-Cormier appartient alors au cercle Élisabeth Dimitriev, un groupe de travail préoccupé par la lutte des classes et faisant partie du MLF. Cet engagement influence la perspective de l'ensemble du collectif de traduction. La conscience de classe est un facteur important dans la tentative du collectif de faire publier la traduction française par une maison d'édition féministe, Les Éditions des femmes, basée à Paris et toujours active de nos jours. Cependant, Les Éditions des femmes refusent de publier Notre corps, nous-mêmes, car elles trouvent que le projet n'est pas assez théorique et qu'il est trop éloigné de leur ligne éditoriale de féminisme radical. De son côté, le collectif de traduction est très déçu et juge que les éditrices sont trop «intellectuelles» et insuffisamment conscientes de la lutte des classes et de la condition des femmes ouvrières ${ }^{11}$. La traduction est finalement publiée par une maison d'édition plus conventionnelle: Albin Michel. Il reste que cet exemple montre à quel point l'expression «nous les femmes» était instable et contestée lorsque les différences entre les femmes nétaient pas prises en compte.

Le processus d'adaptation de $O B O S$ en français a amené les membres du collectif de traduction à s'engager dans une réflexion sur leur propre identité. Cela signifiait de se questionner sur qui elles étaient par rapport à leurs lectrices, mais aussi par rapport aux femmes américaines du collectif de Boston. Afin d'adapter les chapitres sur la santé et le corps des femmes, le collectif français a

10. Par exemple, le chapitre sur l'alimentation a été rédigé par le collectif de traduction. De plus, chaque chapitre comprend une liste de sources locales utiles, et la plupart des photos qui figurent dans l'ouvrage ont été prises par le collectif: des photos des membres du collectif elles-mêmes, de femmes françaises ou de manifestations dans les rues de Paris.

11. Entretien avec Nicole Bizos-Cormier et Sophie Mayoux, septembre 2015. 
dû chercher sa propre voix. Dans ce contexte, «nous les femmes» du collectif de Boston et «nous les Américaines» devient «nous les femmes» du collectif de traduction et «nous les Françaises». La réflexion des traductrices sur leur identité et la façon de représenter les perspectives des femmes illustre leur approche de la réflexivité et leur recherche de leur voix propre. L'acte de traduire comportait un processus de production des savoirs. En effet, comme l'a mis en évidence Kathy Davis, OBOS amène les lectrices à se positionner par rapport au texte. Par extension, quand il s'agit de traduction, l'interaction avec le texte est portée à un niveau supérieur : non seulement le texte interpelle les traductrices sur leurs propres réalités expérientielles, mais la création d'un nouveau texte contextualisé les amène à remettre en question les pratiques locales et les faits relatifs à leur propre contexte social, politique et historique. Ce travail exigeait également que les traductrices reconnaissent leur place dans les rapports de pouvoir caractéristiques du processus de production des savoirs. Qui plus est, cette approche illustrait bien la pratique de réflexivité qui, dans les années 1970, sous l'influence de la recherche féministe, gagnait de l'importance dans des disciplines comme la sociologie et l'anthropologie (Hertz, 1997). Selon cette approche, la chercheure a la responsabilité d'évaluer sa propre posture vis-à-vis des sujets de sa recherche afin de composer avec les différences et l'asymétrie du pouvoir qui découlent des différences de statuts et de parcours de vie (Wasserfall, 1997). Ainsi, la réflexivité implique «a continued self-awareness about the ongoing relationship between a researcher and informants " (ibid., p. 151).

De la même façon, lorsque les membres du collectif français se rendent compte que traduire $O B O S$ mot à mot produit un texte sans voix propre et qui ne s'adresse pas directement aux femmes françaises, elles adoptent une approche réflexive dans le but de créer une adaptation qui reflète la multiplicité des voix. Comme Kate Sturge le suggère, la réflexivité souligne les conditions dans lesquelles se trouve le texte cible, et l'analyse de ces conditions est essentielle pour comprendre comment l'acte de traduire transforme les savoirs. Elle écrit à ce sujet : «Reflexivity accepts the perspectival nature of knowledge and that knowledge is therefore bound to change in transit between languages" (Sturge, 2007, p. 66). Le contexte français des manifestations de mai 1968, y compris le militantisme du MLF de l'époque, 
forme les conditions dans lesquelles les lectrices françaises peuvent interpréter Notre corps, nous-mêmes et ses messages féministes revendicateurs d'autonomie et d'émancipation. «Nous les femmes» dans la traduction française avait la responsabilité d'incorporer non seulement le «nous» des femmes américaines, mais la pluralité des voix exprimées dans le texte.

Les quelques exemples qui suivent illustrent comment la réflexivité a été mobilisée dans la traduction française afin de déterminer la position du collectif de traduction vis-à-vis des lectrices françaises et des auteures du texte source. Le premier exemple est tiré de la préface; les traductrices y discutent ouvertement de leur identité, de leur approche du processus de traduction et de ce qu'elles espèrent que leur version de $O B O S$ accomplira. Au sujet du contenu préfaciel, la spécialiste en traduction féministe, Emek Ergun, observe notamment :

preface writing [...] [is] an invaluable ethical practice for establishing such crosscultural contact zones between feminists since the feminist translator's self-reflexivity about her textual strategies and signature not only discloses her ideological stance to the readers but also serves to expose cross-border interconnectedness among feminists. (2015, p. 72)

Selon ce principe, une préface réflexive facilite les conversations transnationales ainsi que le partage des savoirs parmi les féministes. La préface de la traduction française de $O B O S$ reconnaît le contexte politique tendu qui domine la France à la fin des années 1960 et durant les années 1970. Les femmes françaises qui lisaient Notre corps, nous-mêmes saisissaient inévitablement la signification des messages féministes dans un contexte marqué par le militantisme féministe, les exigences d'égalité et les manifestations récurrentes. Le «nous» employé dans la préface est défini de la manière suivante :

Nous avons entre 24 et 30 ans; l'une d'entre nous est canadienne anglophone; nous avons toutes été jusqu'en faculté, mais nos gagne-pain n'ont souvent rien de prestigieux (secrétaire, vacataire, éducatrice, vendeuse, infirmière auxiliaire, professeur audio-visuel). Deux d'entre nous terminent leurs études. Nous avons toutes milité dans le Mouvement de Libération des Femmes, quelques-unes dans l'extrême gauche. (Collectif de Boston pour la santé des femmes, 1977, p. 10) 
Le ton de confidences adopté par les traductrices françaises reproduit la pratique réflexive des auteures américaines, qui écrivent : "We are white middle-class women, and as such can describe only what life has been for us» (BWHBC, 1973, p. 2).

Les exemples qui suivent mettent pour leur part en évidence le «nous» changeant dans la traduction française, qui permet de percevoir un glissement de sens ainsi que des positions subjectives différentes dans le déplacement d'un contexte à l'autre. Le «nous » utilisé dans les deux extraits ci-dessous représente les membres du collectif de Boston :

Nous pensons que les défenseurs et les adversaires de la pilule sont également à prendre en considération. Après avoir recueilli leurs avis, nous avons pensé de la sorte [...]. (Collectif de Boston pour la santé des femmes, 1977, p. 113).

Voici ce que nous écrit une femme [...]. (ibid.).

Ce sont les membres du collectif de Boston - et non les traductrices - qui ont reçu les lettres des lectrices et qui se sont formé une opinion sur la pilule. Plus loin dans la version française de $O B O S$, les lectrices peuvent entendre la voix des membres du collectif de traduction :

Si vous avez des problèmes, le mieux est de prendre contact avec les organisations (M.L.A.C, G.I.S., Choisir) de votre ville dont nous donnons les adresses dans le chapitre sur la contraception. (ibid., p. 140).

La mention des groupes locaux et d'autres détails lève toute ambiguïté : c'est la voix des traductrices françaises qui se fait alors entendre. Puis, dans la phrase suivante, on note un effort d'inclure une pluralité de voix et de situations qui représentent les conditions diverses dans lesquelles vivent les femmes:

Ainsi, nous préférons parfois attendre un certain temps avant d'avoir des enfants, ou nous nous décidons à ne pas en avoir; nous nous heurtons alors à beaucoup d'incompréhension, mais l'opinion des autres ne doit pas déterminer notre vie. (ibid., p. 158)

La traduction française de $O B O S$ devient un tissage de voix, à savoir le «nous» des féministes de Boston, le «nous» des femmes en général, réclamant une identité partagée des femmes, et le 
«nous» des traductrices françaises. Par conséquent, il est parfois difficile de distinguer quel «nous» prend la parole.

À sa publication, Notre corps, nous-mêmes a eu du succès auprès des femmes en France ainsi que dans les pays francophones européens comme la Belgique et la Suisse, où bon nombre de groupes féministes œuvraient alors à créer un réseau féministe (Nissim, 2014; Pereira, 2015). La traduction s'est bien vendue, et une édition révisée par un autre groupe de militantes féministes françaises est parue en 1981. Au fil du temps, Notre corps, nousmêmes, tout comme le OBOS américain, a toutefois perdu son caractère révolutionnaire compte tenu de la publication croissante d'autres ouvrages consacrés à la santé des femmes.

\section{Une version en français en Afrique de l'Ouest}

En 2004, presque trente ans après la publication de la première version française de $O B O S$, une deuxième version paraît dans cette langue, dans un contexte complètement différent. Intitulée Notre corps, notre santé. La santé et la sexualité des femmes en Afrique subsaharienne, cette version voit le jour au Sénégal, à l'initiative de Codou Bop, chercheure et féministe, et Fatou Sow, sociologue et professeure à l'Université Cheikh Anta Diop de Dakar dont la recherche et les travaux universitaires ont fait une contribution importante à la visibilisation de la participation et des mouvements sociaux des femmes sénégalaises. Bop et Sow sont également des militantes des droits des femmes de longue date et des figures emblématiques du mouvement féministe africain ancré dans la réalité sénégalaise. C'est la maison d'édition L'Harmattan qui assure la publication de cette version.

En marge d'un congrès de Recherches Féministes Francophones tenu à Montréal en 2015, Codou Bop explique que le livre original a servi essentiellement d'inspiration au projet sénégalais. Bop et Sow ne sont pas parties du texte américain, mais plutôt de leurs présuppositions de ce que des femmes sénégalaises souhaitaient savoir sur la santé et la sexualité. Elles ont soumis leurs questions au milieu médical et ont rédigé les réponses dans un français accessible et vulgarisé. Puis, dans le cadre du Réseau de recherche en santé de la reproduction en Afrique francophone (RRSRAF), elles ont réuni une dizaine de chercheures féministes de la région ouest-africaine afin de valider et de bonifier le texte avant sa publication. 
Dans la préface de louvrage, Bop et Sow font preuve de réflexivité en exposant le processus d'élaboration du livre. En effet, elles soulignent que ce sont des experts et des expertes qui l'ont écrit : des médecins, des sociologues, des biologistes, etc. Les éditrices sénégalaises révèlent que les préoccupations féministes portées par certaines des expertes ont dû se confronter au conservatisme de certaines autres auteures pour faire exister un texte vulgarisé, accessible et proche des intérêts des femmes. Elles attirent également l'attention sur la difficulté de s'adresser à des femmes d'Afrique de l'Ouest : la multiplicité des langues parlées dans la région et le fait que ces langues soient pour la plupart de tradition orale ont mené les éditrices à choisir le français comme langue de publication. Les conséquences de la colonisation de la région, bien qu'elles ne soient pas nommées comme telles dans la préface, se font donc encore sentir près de quarante ans après les indépendances. Il s'ensuit que cette version française de $O B O S$ s'adresse plutôt aux intervenants et intervenantes du milieu de la santé et au personnel des ONG ou des associations de la société civile, afin de les inviter à agir.

L'analyse du texte révèle que l'utilisation du «nous» (ou de «notre» et «nos») diverge de ce qu'on observe dans la version française de 1977. De fait, le pronom et ses dérivés apparaissent très rarement dans le texte sénégalais: moins d'une vingtaine d'occurrences dans un livre de plus de 300 pages. Ils désignent les Sénégalais et les Sénégalaises en général, comme dans l'extrait suivant: "Il est [...] important de mettre l'accent sur le potentiel extraordinaire que représentent les adolescents, avenir de notre continent» (RRSRAF, 2004, chap. Adolescente; nous soulignons) ${ }^{12}$. Quand il s'agit de traiter des expériences et de la santé des femmes, les auteures ont choisi d'utiliser des expressions telles que «la femme» ou «les femmes»: «la femme veuve», «la jeune femme», «la femme divorcée» ou «les femmes enceintes», «les femmes africaines». Par exemple, on peut lire : «Les méthodes de barrière sont peu coûteuses, n'affectent en rien le fonctionnement du corps de l'homme ou de la femme, et ont très peu d'effets secondaires, même si la femme allaite» (RRSRAF, 2004, chap. Planning familial; nous soulignons).

12. Toutes les citations sont tirées des chapitres disponibles sur le site de la Our Bodies Ourselves Global Initiative (http://www.ourbodiesourselves.org/ global-projects/senegal-research-group-on-women-and-laws-in-senegal/). 
On trouve par ailleurs le pronom possessif «notre» dans l'un des rares témoignages de l'ouvrage. "[Mon mari] a commencé à me battre à peine un mois après notre mariage et il a continué» (RRSRAF, 2004, chap. Violence; nous soulignons). Il ne désigne ici ni les femmes en général ni les féministes, mais l'expérience spécifique de la femme qui témoigne. Finalement, les auteures l'utilisent pour désigner une de leurs informatrices dans un encadré où elles rapportent les propos tenus pendant une entrevue. "Notre informatrice explique que, lorsqu'on masse les bébés, on peut vérifier dans une certaine mesure si l'enfant est bien formé ou non» (RRSRAF, 2004, chap. Accouchement et période postnatale; nous soulignons). Le pronom possessif réfère donc cette fois aux auteures elles-mêmes.

Il n'y a en fin de compte que dans le titre du livre, Notre corps, notre santé, que le pronom possessif de la troisième personne du pluriel désigne l'ensemble des femmes et prend le sens de "nous les femmes». Et même là, le sous-titre - La santé et la sexualité des femmes en Afrique subsaharienne -, avec la formulation "des femmes", recrée une certaine distance. Les auteures auraientelles adopté ce titre uniquement pour faire écho à l'ouvrage qui les a inspirées? Tout porte à le croire puisqu'ailleurs dans leur livre, "notre» s'applique généralement aux Sénégalais et Sénégalaises. Ancrée dans un contexte africain bien défini, l'adaptation reconnaît le rôle clé des cultures africaines dans la lutte féministe au Sénégal. Or, même sans le «nous», le travail académique et le militantisme de Bop et Sow et leur projet d'adaptation de $O B O S$ pour les femmes sénégalaises ont apporté une contribution très importante aux mouvements sociaux et à la prise de conscience des femmes au Sénégal.

\section{Une version en français au Québec}

Contrairement aux cas précédents, la version québécoise de $O B O S$ n'existe pas encore. Elle est en cours d'élaboration, et l'analyse ne pourra pas porter sur le texte final, mais se concentrera sur le processus. Afin de mieux l'appréhender, nous commencerons par tracer un portrait succinct du développement de la théorie intersectionnelle aux États-Unis et nous verrons comment cette théorie est mise en application dans le domaine de la santé sexuelle et reproductive, et plus particulièrement dans la version américaine de OBOS de 2011. Puis nous traiterons de la diffusion et de la 
pratique de l'intersectionnalité au Québec. Nous verrons que la mise en application de cette théorie amène les initiatrices de la version québécoise à se questionner sur l'usage du "nous-femmes » et à chercher des solutions à la représentation de la diversité des identités, des vécus et des opinions des femmes.

\subsection{Théorie et pratique de l'intersectionnalité aux États-Unis}

Aux États-Unis, dès les années 1960 et 1970, des féministes afro-américaines comme bell hooks ${ }^{13}$, Angela Davis et Audre Lorde traitent de la complexité des oppressions vécues quand on est femme, noire et lesbienne. À la fin des années 1980, Kimberlé Crenshaw propose un concept pour analyser la diversité des identités et des oppressions qui leur sont associées : l'intersectionnalité. Cette avocate afro-américaine montre que les femmes noires sont doublement réduites au silence : le racisme les rend invisibles dans les discours des féministes blanches et le sexisme en fait autant dans les mouvements antiracistes afroaméricains.

Le problème avec la politique de l'identité n'est pas qu'elle échoue à transcender la différence comme l'en accusent certains critiques, mais plutôt l'inverse : la plupart du temps, elle amalgame ou ignore les différences internes à tel ou tel groupe. (Crenshaw, 2005 [1991], p. 53)

Avec le concept d'intersectionnalité, Crenshaw met en évidence les différences qui existent entre les femmes. En remettant en cause l'idée d'un vécu unanimement partagé, elle réfute la pertinence d'un «nous les femmes».

Les auteurs de textes féministes se trouvent alors devant un questionnement face à l'usage d'une terminologie englobante qui laisserait sous-entendre que toutes les femmes vivent une expérience commune. Comment les femmes peuvent-elles dorénavant utiliser le «nous»? Quel effet peut avoir l'adoption d'une approche intersectionnelle sur l'écriture, au niveau du processus et du résultat? C'est à ces questions que nous, les auteures du présent article, cherchons à répondre par l'étude de $O B O S$ et de ses traductions francophones.

Depuis Crenshaw, le concept d'intersectionnalité a été repris dans nombre de recherches qui abordent la multiplicité et l'imbrication des systèmes d'oppression (patriarcat, colonialisme,

13. Nom de plume de Gloria Jean Watkins, qu'elle écrivait sans majuscules. 
racisme, néolibéralisme, capacitisme, etc.). À la fois concept, théorie et approche, l'intersectionnalité gagne en popularité, entre autres grâce au caractère flou de sa définition et à la flexibilité de son affiliation épistémologique (Davis, 2015). Il reste que, à vouloir tout englober, l'intersectionnalité risque de ne rien englober du tout et, pire encore, de ne plus remplir le rôle pour lequel elle a été créée : remettre en question l'hégémonie du féminisme blanc de classe moyenne. Les critiques féministes lesbiennes, noires ou colonisées portées au féminisme «blanc de classe moyenne» ne sont plus perçues que comme un exemple de pensée intersectionnelle parmi d'autres (Carbin et Edenheim, 2013, p. 236). Certaines se questionnent même sur «le blanchiment de l'intersectionnalité» :

[II] y a lieu de se demander si l'intersectionnalité ne serait pas devenue cette version de la pensée féministe noire qui s'épanouit dans le monde universitaire sans toutefois une participation significative des femmes racialisées ou si le processus d'institutionnalisation de l'intersectionnalité ne conduirait pas à leur marginalisation et à leur effacement. (Bilge, 2015, p. 9)

Dans une perspective plus pratique et dans le domaine de la santé sexuelle et reproductive, l'intersectionnalité se matérialise par le développement de l'action pour la justice reproductive. Aux États-Unis, des groupes de femmes racisées, en situation de handicap ou emprisonnées, signalent que les instances médicales, gouvernementales et juridiques n'exercent pas leur pouvoir de la même façon selon qu'elles ont affaire à une femme blanche hétérosexuelle de classe moyenne ou à une femme qui vit à l'intersection de multiples oppressions. Par exemple, des groupes comme SisterSong, Black Women for Reproductive Justice, The Pro-choice Public Education Project ou Asian Communities for Reproductive Justice font valoir que si le patriarcat exige des femmes blanches qu'elles reproduisent la population active, le système carcéral et le système médical ont plutôt tendance à décourager la maternité des femmes racisées ou en situation de handicap.

On peut sans doute considérer que la neuvième et dernière édition de $O B O S$ (2011) s'inscrit dans la mise en application de l'approche intersectionnelle, dans la mesure où la diversité y est plus que jamais à l'avant-plan. Dans les premières éditions, les identités des femmes qui élaborent le livre et qui y sont représentées sont assez 
homogènes : blanches, relativement éduquées, hétérosexuelles et de classe moyenne. Ces femmes sont conscientes de l'homogénéité de leurs identités et font preuve de réflexivité en la nommant dès les premières pages du livre. Puis, au fil des ans et des rééditions, $O B O S$ a su élargir son réseau et intégrer une multiplicité de voix. On trouve ainsi les témoignages de femmes de tous âges, qu'elles soient Latinas, afro-américaines, d'ascendance anglo-saxonne ou immigrantes, lesbiennes, hétérosexuelles, bisexuelles, asexuelles, queer, cis, trans ou intersexuelles. Le chapitre 5 de la dernière édition, «Relationships» (BWHBC, 2011), est d'ailleurs consacré à la retranscription d'une discussion en ligne entre 36 femmes aux identités diverses, de l'hétérosexuelle de 24 ans originaire de Corée à la bisexuelle de 63 ans de descendance anglo-saxonne et tout juste sortie d'un ménage à trois.

Les pronoms «we», «our» et «us» y sont abondamment utilisés dans le sens de "nous les femmes». Par exemple, on peut lire «Understanding the social and personal influences on our sexuality can help us claim our right to pleasure» (BWHBC, 2011, p. 141; nous soulignons). Cependant, ce «nous» reste inclusif et s'applique autant à des identités minoritaires que majoritaires. Ainsi, on traite de "nos handicaps» ou "notre religion ou notre absence de religion». "We must also decide when and how much to disclose if our disability is not obvious [...]» (BWHBC, 2011, p. 190; nous soulignons).

La collective de militantes qui traduit et adapte $O B O S$ en français au Québec part de cette dernière édition américaine. La Collective pour un ouvrage de référence participatif sur la santé féministe, la CORPS féministe, s'est formée à l'initiative de militantes et de travailleuses de groupes de défense des droits des femmes. Elle est composée de dix femmes cis âgées de 28 à 63 ans dont trois immigrantes de première génération originaire d'Europe et d'Afrique du Nord et deux immigrantes de deuxième génération originaires d'Europe du Sud et d'Amérique latine. Six d'entre elles sont hétérosexuelles et quatre s'identifient comme bisexuelle, pansexuelle ou queer. Quatre ont des enfants - âgés de 9 à 36 ans - dont une qui a deux enfants vivant avec un trouble $\mathrm{du}$ spectre de l'autisme. Une des membres est en situation de handicap et deux s'identifient comme "femmes voluptueuses». Six ont le français comme langue maternelle et quatre ont d'abord appris l'anglais ou une autre langue (arabe, espagnol, italien). 
La CORPS féministe approche la traduction avec beaucoup de liberté face à l'original. En effet, ce qui importe à ses membres n'est pas uniquement de faire voyager un texte et l'information qu'il contient : elles cherchent à faire résonner un processus qui sert autant la production de savoirs féministes sur le corps que la création de liens entre des groupes de femmes qui ne se côtoient pas suffisamment. Comme le mentionne Kathy Davis à propos de l'épopée transnationale de $O B O S$,

[t]he translators adopted this process of collective knowledge making, using their translations to generate similar discussions among women in their own context. It is this feature of $O B O S$ - more than its informational content, its format, or its specific feminist politics of health-that ultimately proved to be universally translatable. (2007, p. 195)

Nous verrons, dans les paragraphes qui suivent, comment le projet de la CORPS féministe prend source dans les dynamiques actuelles du mouvement des femmes au Québec et se caractérise par la volonté d'une mise en pratique de l'intersectionnalité.

\subsection{Les groupes de femmes au Québec}

Le mouvement des femmes au Québec a consolidé son élan des années 1960-1970 en mettant sur pied des groupes de défense des droits des femmes jusque dans les années 1980. Ainsi, la Fédération des femmes du Québec (FFQ) est fondée en 1966, la Fédération du Québec pour le planning des naissances (FQPN) en 1972, le Regroupement des maisons d'hébergement pour femmes victimes de violence en 1975, etc. Ces groupes et réseaux se caractérisent par le fait qu'ils ont été créés par et pour des femmes, dans leurs communautés et dans une perspective d'entraide. Plusieurs d'entre eux utilisent des pratiques de conscientisation pour développer leur analyse et encourager la solidarité entre femmes issues de diverses classes sociales. Ils visent «à créer des espaces où des prises de conscience pourront s'opérer au vu du caractère systémique des oppressions» (Bessaih, 2013, p. 132).

Dès la fin des années 1980, des chercheures attirent l'attention sur le fait que ces organismes restent majoritairement animés par des femmes blanches, francophones et québécoises depuis plusieurs générations (Belleau, 1996; Chouakri, 2001; Osmani, 2002). Les années 2000 s'accompagnent de l'éclatement 
de la notion de genre, de l'émergence d'un mouvement pour la sexualité positive, de mobilisations des femmes autochtones et d'une prise de parole publique des personnes en situation de handicap. Ces événements et ces phénomènes remettent en question l'homogénéité des groupes de femmes au Québec et la primauté accordée aux enjeux liés à l'égalité hommes-femmes (Marchand et Corbeil, 2006; Mensah, 2009; Masson, 2013; Pagé, 2014; Maillé, 2014). Cependant, les personnes et les groupes qui développent un discours public sur les multiples oppressions vécues par des femmes demeurent en périphérie des groupes de femmes blanches qui existent depuis plus de quarante ans (FFQ, FQPN, etc.). Par ailleurs, malgré la traduction et la circulation de textes sur l'intersectionnalité, il apparaît que les féministes de langue française n'ont pas adopté ce concept aussi nettement que leurs vis-à-vis anglophones. "La question posée en 1988 par [Gayatri Chakravorty] Spivak - Can the Subaltern Speak? - n'a pas eu la même résonance dans les féminismes de la francophonie et dans les féminismes qui circulent en anglais» (Maillé, 2014, p. 53).

Si la théorie intersectionnelle fait son chemin dans le milieu universitaire francophone, avec les lacunes que l'on a exposées, sa mise en pratique se fait encore attendre. "La question des différences entre les femmes et la prise de parole des femmes de la périphérie a ouvert la voie à un fantastique renouvellement de l'analyse féministe, mais comment traduire ces analyses en termes organisationnels pour les mouvements de femmes?»(Maillé, 2014, p. 56).

\subsection{Tenir compte de la diversité des identités des femmes dans le projet de la CORPS féministe}

$\mathrm{Au}$ Québec, l'intersectionnalité fait parler d'elle sur le plan théorique, mais elle tarde à se manifester dans la pratique. C'est dans ce contexte que la CORPS féministe entreprend la traduction et l'adaptation de OBOS. Sur son site Web, elle témoigne de son affiliation avec l'intersectionnalité en affirmant :

Notre approche se positionne dans une perspective féministe, anticoloniale, antiraciste et non hétéronormative. Cela signifie que nous reconnaissons diverses oppressions à l’ouvre dans notre société. (https://lacorpsfeministe.org/)

Ses membres connaissent la pratique des groupes de conscientisation qui existe au Québec depuis plusieurs décennies 
et qui a contribué à forger une analyse féministe qui tient compte des rapports de genre et de classe. Parallèlement, l'effervescence de l'approche intersectionnelle dans le milieu universitaire coïncide avec le moment où les groupes de femmes établis de longue date entendent enfin la voix des femmes les plus marginalisées (racisées, trans, en situation de handicap, etc.). Quand les membres de la CORPS féministe lisent $O B O S$, elles y reconnaissent une pratique de partage des vécus et de mise en commun des expériences qui existe déjà au Québec, mais qui, dans l'ouvrage américain, a le mérite d'inclure les femmes les plus marginalisées. Elles ne cherchent alors pas à traduire le texte mot pour mot. Elles souhaitent faire écho au processus qui sous-tend l'élaboration du livre et s'inscrivent donc dans la continuité des pratiques de conscientisation qu'elles tentent maintenant d'appliquer en tenant compte des analyses intersectionnelles. On est ici face à une démarche d'adaptation culturelle qui s'autorise volontairement l'infidélité face au texte source, mais qui se place plutôt en écho au processus sous-jacent de production de savoirs féministes.

La CORPS féministe tente de bâtir un pont entre les promesses théoriques de l'intersectionnalité et un travail politique de terrain au Québec. Faire atterrir la théorie intersectionnelle dans la pratique est aussi une façon de ne pas s'égarer dans les zones floues de l'intersectionnalité qui ont été dénoncées par certaines chercheures (Carbin et Edenheim, 2013; Davis, 2015). L'intersectionnalité est alors signifiante à deux niveaux : au niveau $\mathrm{du}$ processus d'élaboration du texte et au niveau de ce que le texte donnera à lire. Ainsi, lorsque la CORPS féministe adopte une approche intersectionnelle, cela implique d'élaborer le texte en co-construction avec des groupes de femmes marginalisées et d'inscrire dans le texte la présence et la parole des femmes les plus marginalisées.

Une des auteures du présent article, membre de la CORPS féministe, se trouve dans une position privilégiée pour observer le processus conçu et mis en œuvre dans le projet. À travers l'observation participante, l'analyse des procès-verbaux de rencontres et les publications diffusées sur le site Web du groupe, nous constatons que la CORPS féministe recourt à la co-construction et à des pratiques d'action sociale militante et communautaire pour développer une prise de parole représentative de la diversité des femmes vivant au Québec. Quatre formes de 
pratiques participatives se dégagent: la diffusion du projet, la constitution d'un comité éditorial et coordonnateur, la collecte de témoignages et la mobilisation des savoirs sociaux et universitaires.

Contrairement à la version sénégalaise de 2004 et à la version française de 1977, l'adaptation de $O B O S$ au Québec prend appui d'abord et avant tout sur un travail de mobilisation visant à faire connaître le projet et son ouverture à la participation de toutes les personnes en accord avec son approche. Cette mobilisation est basée sur des méthodes d'action sociale déjà pratiquées au Québec comme les appels à participation et l'organisation de groupes de discussions. Elle recourt également à des considérations que l'on pourrait qualifier d'éthiques et qui se rattachent à l'approche intersectionnelle. Ainsi, les procès-verbaux du comité éditorial et coordonnateur de la CORPS féministe montrent que le groupe consacre du temps à réfléchir et à développer des moyens pour tenir compte de la diversité des femmes et des oppressions qu'elles vivent. À titre d'exemple, on trouve les points suivants dans les ordres du jour : "Comment renforcer et mettre en pratique notre compréhension de l'intersectionnalité? », «Inclusion des personnes non binaires", "Que faire dans le livre vis-à-vis des opinions politiques divergentes dans le mouvement des femmes?", etc. On y traite aussi de sujets administratifs, logistiques et rédactionnels, dont certains illustrent un souci d'inclusion : «Féminisation et usage des termes épicènes", "Changement du nom "CORPS des femmes» pour "CORPS féministe». Lors d'une rencontre de travail sur l'un des chapitres, une discussion s'est tenue sur l'usage du «nous » dans le texte. Il a été établi que ce pronom serait réservé à désigner les membres de la CORPS féministe, comme dans la phrase suivante : "Ainsi, nous avons accordé une vigilance particulière à garder un texte ouvert, qui ne limite pas le contenu à une seule expérience» (chapitre 7 en cours de rédaction; nous soulignons).

À partir de questions tirées du livre original, la CORPS féministe a dressé une liste de questions dont les réponses constitueront des témoignages pour la version québécoise. Sur son site, elle offre un outil de collecte de témoignages et dans ses actions, elle multiplie les moyens d'aller chercher la parole des femmes les plus marginalisées. Puis, dans un souci d'inclusion et d'élargissement de ses perspectives, elle s'ouvre aux commentaires de groupes et de collectifs de femmes marginalisées. Elle les 
invite à s'approprier les questions, en les complétant, en les modifiant et en déterminant si et comment leurs membres souhaiteraient y répondre (entrevues individuelles ou de groupes, questionnaires anonymes, forums de discussions, etc.). Par cette approche, les groupes participent à définir le contenu du livre et plus particulièrement des témoignages. Notons que la CORPS féministe ne cherche pas à être statistiquement représentative de la population du Québec : elle cherche à représenter la diversité en s'assurant qu'une multiplicité de «savoirs situés» (Haraway, 1988) s'exprime dans les témoignages.

La CORPS féministe implique donc des groupes dans la collecte de témoignages et dans la définition de sujets qui seront abordés dans le livre. Elle projette aussi d'intégrer du contenu critique produit par des groupes ou par des recherches en sciences sociales ou en sciences de la santé. De plus, dans son plan d'action, on peut lire qu'une des dernières étapes consistera à soumettre son texte final aux commentaires et aux bonifications de personnes identifiées comme des militantes pour la justice reproductive ou des chercheures féministes et critiques dans le domaine de la santé ou des sciences sociales. Ces personnes seront conviées à une rencontre pour délibérer sur leurs commentaires. En co-construisant le contenu du livre, la CORPS féministe vise ainsi à créer un espace d'action commune pour des personnes et des groupes intéressés par la justice reproductive. Cette action commune constitue une occasion de développer des liens et de renforcer un mouvement par la diversification et l'inclusion.

\section{Conclusion}

Malgré les contextes très variés, des États-Unis des années 1970 à la France post-mai 1968, au Sénégal des années 2000 et au Québec d'aujourd'hui, $O B O S$ continue d'inspirer le partage des savoirs sur la santé reproductive et la sexualité des femmes. Ces sujets, même s'ils sont moins tabous qu'à d'autres époques, restent rarement discutés ouvertement dans des termes qui n'évoquent pas la honte. De surcroît, la diversité des expériences féminines dans le domaine de la santé reproductive et de la sexualité contribue à complexifier les échanges. Comme nous l'avons vu, au fil des années, les auteures et les traductrices de OBOS ont œuvré à reconnaître les voix diverses qui constituent le «nous les femmes» en recourant à la réflexivité et l'intersectionnalité. Au 
Québec, la méthode de co-construction des savoirs, et donc du contenu du livre, recèle un potentiel d'élargissement de la notion de fidélité au texte source et entraîne une réflexion approfondie et concrète sur un projet politique féministe et inclusif. Le choix de la co-construction dans le projet de traduction et d'adaptation entrepris par la CORPS féministe est explicitement lié à la théorie intersectionnelle et constitue un exemple de sa mise en œuvre. Tout comme la réflexivité dans les années 1970, l'intersectionnalité répond à une nécessité qui perdure dans les années 2010 de représenter les femmes dans le respect de leurs identités diverses. Finalement, les traductions de $O B O S$ rappellent que l'expression «nous les femmes» doit tenir compte de la diversité si elle veut prétendre à renforcer le mouvement féministe et ses futurs projets d'émancipation.

\section{Références}

Belleau, Josée (1996). «Le féminisme "tricoté serré" en question: perspectives de Québécoises de minorités ethnoculturelles». Cabiers de recherche du GREMF, 74, p. 155-166.

Bessaïh, Nesrine (2013). «L'R des centres de femmes du Québec». Nouvelles questions féministes, 32, 2, p. 130-134.

Bilge, Sirma (2015). «Le blanchiment de l'intersectionnalité». Recherches féministes, 28, 2, p. 9-32.

Boston Women's Health Book Collective (BWHBC) (2011 [1971, 1973, 1976, 1979, 1984, 1992, 1998, 2005]). Our Bodies, Ourselves. New York, Simon \& Schuster.

Bracke, Maud Anne (2015). «Our Bodies, Ourselves: The Transnational Connections of 1970s Italian and Roman Feminism». Journal of Contemporary History, 50, 3, p. 560-580.

Brisset, Annie (1990). Sociocritique de la traduction. Longueuil, Le Préambule.

Carbin, Maria et Sara Edenheim (2013). «The Intersectional Turn in Feminist Theory: A Dream of a Common Language?». European Journal of Women's Studies, 20, 3, p. 233-248.

Castro, Olga et Emek Ergun (2017). Feminist Translation Studies: Local and Transnational Perspectives. New York, Routledge.

Chamberlain,Lori (1988). «Gender and the Metaphorics of Translation». Signs: Journal of Women in Culture and Society, 13, 3, p. 454-472.

Chouakri, Yasmina (2001). Les femmes et la diversité ethnoculturelle à Montréal, ou Comment la Table des groupes de femmes de Montréal peut-elle faire une place aux femmes et aux groupes de femmes de diverses 
origines et intégrer leurs préoccupations? : une recherche-action. Montréal, Table des groupes de femmes de Montréal.

Collectif de Boston pour la santé des femmes (1977). Notre corps, nousmêmes. Trad. Collectif français des adaptatrices. Paris, Albin Michel.

Crenshaw, Kimberlé (2005 [1991]). "Cartographie des marges: intersectionnalité, politique de l'identité et violence contre les femmes de couleur». Trad. Oristelle Bonis. Cabier du genre, 2, 39, p. 51-82.

Davis, Kathy (2007). The Making of Our Bodies, Ourselves. How Feminism Travels across Borders. Durham/London, Duke University Press.

Davis, Kathy (2015). «L'intersectionnalité, un mot à la mode. Ce qui fait le succès d'une théorie féministe». Trad. Françoise Bouillot. Les Cahiers du CEDREF, 20. Disponible à : <http://cedref.revues. org/827> [consulté le 2 mai 2017].

Ergun,Emek (2015).Doing Feminist Translation as Local and Transnational Activism. The Turkish Translation of Virgin: The Untouched History and Its Reception. Thèse de doctorat. University of Maryland. Inédit.

Fassin, Éric (2015). «D'un langage l'autre : l'intersectionnalité comme traduction». Raisons politiques, 58, 2, p. 9-24.

Federici, Eleonora, dir. (2011). Translating Gender. Berne, Peter Lang.

Godard, Barbara (1989). «Theorizing Feminist Discourse/Translation». Tessera, 6, p. 42-53.

Gordon, Linda et Berrie Thorne (1998). «Feminist Subversions: Our Bodies, Ourselves». In D. Clawson, dir. Required Reading: Sociology's Most Influential Books. Amherst, University of Massachusetts Press, p. 181-188.

Haraway, Donna (1988). «Situated Knowledges: The Science Question in Feminism and the Privilege of Partial Perspective». Feminist Studies, 14, 3, p. 575-599.

Hertz, Rosanna, dir. (1997). Reflexivity and Voice. Thousand Oaks [Californie], Sage.

Kramarae, Cheris et Dale Spender, dir. (2000). Routledge International Encyclopedia of Women: Global Women's Issues and Knowledge. New York, Routledge.

Lotbinière-Harwood, Susanne de (1991). Re-belle et infidèle : la traduction comme pratique de réécriture au féminin = The body bilingual : translation as a re-writing in the feminine. Montréal, Les Éditions du remueménage.

Maier, Carol (1985). «A Woman in Translation, Reflecting». Translation Review, 17, 1 p. 4-8.

Maillé, Chantal (2014). «Approche intersectionnelle, théorie postcoloniale et question de différence dans les féminismes anglo- 
saxons et francophones». Politique et sociétés, 33, 1, p. 41-60.

Marchand, Isabelle et Christine Corbeil (2006). «Penser l'intervention féministe à l'aune de l'approche intersectionnelle : défis et enjeux». Nourvelles pratiques sociales, 19, 1, $\mathrm{p}$ 40-57.

Masson, Dominique (2013). «Femmes et handicap».Recherches féministes, 26, 1, p. 111-129.

Mensah, Maria Nengeh (2009). «Sexe, médias et... hypermoralisation». Globe: Images et représentation de la sexualité au Québec, 12, 2, p. 169180.

Morgen, Sandra (2002). Into Our Own Hands: The Women's Health Movement in the United States, 1969-1990. New Brunswick [NJ], Rutgers University Press.

Nissim, Rina (2014). Une sorcière des temps modernes: le self-help et le mouvement femmes et santé. Genève, Éditions Mamamélis.

Osmani, Farida (2002). «L'égalité pour toutes? L'engagement féministe et les droits des immigrantes au Québec». Recherches féministes, 15, 2, p. 141-151.

Our Bodies Ourselves (s.d.). «OBOS Timeline: 1969-Present». Our Bodies Ourselves. Disponible à : <https://www.ourbodiesourselves. org/our-story/history/obos-timeline-1969-present/> [consulté le 2 mai 2017].

Pagé, Geneviève (2014). «Sur l'indivisibilité de la justice sociale ou Pourquoi le mouvement féministe québécois ne peut faire l'économie d'une analyse intersectionnelle». Nouvelles pratiques sociales, 26, 2, p. 200-217.

Pereira, Sophie (2015). "Notre corps, nous-mêmes». Analyse, 19. Disponible à : <http://www.universitedesfemmes.be/se-documenter/ telechargement-des-etudes-et-analyses/product/200-notre-corpsnous-memes> [consulté le 30 mai 2017].

Pisan, Annie de et Anne Tristan (1977). Histoires du M.L.F. Paris, Calmann-Lévy.

Réseau de recherche en santé de la reproduction en Afrique francophone (RRSRAF) (2004). Notre corps, notre santé. La santé et la sexualité des femmes en Afrique subsaharienne. Paris, L'Harmattan.

Sanford, Wendy Coppedge (1979). «Working Together, Growing Together: A Brief History of the Boston Women's Health Book Collective». Heresies, 2, 3, p. 83-92.

Shapiro, Ester (2013). "Translating Latin American/US Latina Frameworks and Methods in Gender and Health Equity: Linking Women's Health Education and Participatory Social Change». International Quarterly of Community Health Education, 34, 1, p. 1936. 
Simon, Sherry (1996). Gender in Translation: Cultural Identity and the Politics of Transmission. New York, Routledge.

Spivak, Gayatri Chakravorty (2004 [1992]). «Politics of Translation». In L. Venuti, dir. The Translation Studies Reader. Londres et New York, Routledge, p. 369-388.

Sturge, Kate (2007). Representing Others: Translation, Ethnography and the Museum. Manchester, St. Jerome.

Susam-Sarajeva, Şebnem (2006). Theories on the Move: Translation's Role in the Travels of Literary Theories. Amsterdam, Rodopi.

von Flotow, Luise (1997). Translation and Gender: Translating in the 'Era of Feminism.' Ottawa, Les Presses de l'Université d'Ottawa.

von Flotow, Luise, dir. (2011). Translating Women. Ottawa, Les Presses de l'Université d'Ottawa.

von Flotow, Luise et Farzanah Farahzad, dir. (2016). Translating Women: Different Voices and New Horizons. New York, Routledge.

Wasserfall, Rahel R. (1997). «Reflexivity, Feminism, Difference». In R. Hertz, dir. Reflexivity and Voice. Thousand Oaks [Californie], Sage, p. 150-168.

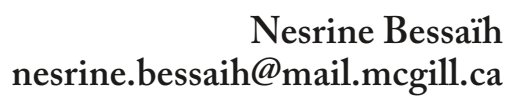

Anna Bogic anna.bogic@gmail.com 